Family Medicine and Community Health

\title{
Obstacles for Iranian rural population to participate in health education programmes: a qualitative study
}

\author{
Yousef Hamidzadeh, ${ }^{1,2}$ Mina Hashemiparast, ${ }^{3}$ Hadi Hassankhani, ${ }^{4}$ \\ Hamid Allahverdipour ${ }^{1,5}$
}

To cite: Hamidzadeh Y, Hashemiparast M, Hassankhani $\mathrm{H}$, et al. Obstacles for Iranian rural population to participate in health education programmes: a qualitative study. Fam Med Com Health 2019;7. doi:10.1136/fmch-2018-000020

Received 15 September 2018 Revised 31 December 2018 Accepted 06 January 2019
Check for updates

(C) Author(s) (or their employer(s)) 2019. Re-use permitted under CC BY-NC. No commercial re-use. See rights and permissions. Published by BMJ.

${ }^{1}$ Candidate in Health Education, Department of Health Education \& Promotion, Tabriz University of Medical Sciences, Tabriz, Iran ${ }^{2}$ Department of Health Education \& Promotion, Tabriz University of Medical Sciences, Tabriz, Iran ${ }^{3}$ Department of Public Health, Maragheh University of Medical Sciences, Maragheh, Iran ${ }^{4}$ Qualitative Research Center, Tabriz University of Medical Sciences, Tabriz, Iran

${ }^{5}$ Research Center for Psychiatry and Behavioral Sciences, Tabriz University of Medical Sciences, Tabriz, Iran

Correspondence to

Professor Hamid Allahverdipour; allahverdipourh@tbzmed.ac.ir

\section{ABSTRACT}

Objective To explore the obstacles of community participation in rural health education programmes from the viewpoints of Iranian rural inhabitants.

Design This was a qualitative study with conventional content analysis approach which was carried out March to October 2016.

Setting Data collected using semistructured interviews that were digitally recorded, transcribed and analysed until data saturation. MAXQDA 10 software was used to manage the textual data.

Participant Participants were twenty-two seven clients from a rural community in Ardabil, Iran who were receiving health services from health centres.

Result The main obstacles to participate in health education programmes in rural settings were 'Lack of trust to the rural health workers', 'Adherence to neighbourhood social networks in seeking health information' and 'Lack of understanding on the importance of health education'.

Conclusion Rural health education programmes in Iran are encountered with a variety of obstacles. We need to enhancing mutual trust between the rural health workers and villagers, and developing community-based education programmes to promote health information seeking behaviours among villagers. The finding of this study will be a referential evidence for the qualitative improvement of local health education programmes for rural inhabitants.

\section{INTRODUCTION}

The WHO has confirmed the shortage of healthcare professionals all over the world, especially in rural areas of developing and low-income countries. ${ }^{1}$ The National Rural Health Care Association (NRHA) has also announced that the obstacles faced by healthcare providers/clients to deliver/ receive healthcare in rural areas are vastly different from those in urban areas. ${ }^{2}$ Literature confirms the failure of health systems in improving the health of various groups in rural regions, regardless of factors like age, gender and race. ${ }^{23}$ Therefore, rural inhabitants of developing countries have still remained in an inappropriate level of health status. ${ }^{4}$ For instance, millions of people in Africa and Asia die from preventable diseases, ${ }^{5}$ while a majority of these diseases could be prevented with health education. $\mathrm{s}^{6}$

Health education is one of the main components of healthcare services in addressing the major health concerns, like maternal and infant mortality, infectious disease and even in healthy life promotion programmes. ${ }^{7}$ This initial cornerstone of primary healthcare services may be well applied in rural areas by community health workers (CHWs), as the health system members who are at the frontline contact with rural populations. ${ }^{8}$ In many rural regions, CHWs are key personnel that support health education programmes for rural and underserved populations. ${ }^{7-9}$ Previous studies have shown that CHWs in developing countries, like Iran, are faced with several challenges in health education of rural communities. ${ }^{10}$ For instances, they lack in ability and skills to deliver health education messages in a consistent manner, which may lead to decrease in their level of productivity and effectiveness in responding to the current health needs of their community. ${ }^{11}$ Such challenges may, consequently, result in a decline in the willingness of rural communities to be approached for receiving health education services. ${ }^{12}$ Previous studies have shown that enough effective health education efforts in rural regions have not been made and also enough evidence on the barriers and challenges of health education activities in such areas is not provided. ${ }^{13} 14$

Literature has indicated various constraints in the ways that the health education programmes are either accessed or delivered. Such constraints mainly address certain aspects of the challenges for CHWs in delivering health education services to the rural communities. $^{14} 15$ However, the number of studies investigating the challenges and obstacles of implementing health education programmes by CHWs in rural and underserved communities are scarce. ${ }^{16}$ Numerous 
obstacles in processes continue to have an adverse impact on the quality of care about failures in patient communication, and patient education was reported in adherence to medication regimes, counselling and community health education and prevention. ${ }^{17}$ Also, to our knowledge, such challenges have not yet been studied from the viewpoints of rural residents. Considering the substantial role of rural health education in improving the knowledge of rural populations on health and promoting their health literacy and eventually health status, there is a great need to identify the obstacles and challenges of health education activities from the viewpoints of rural residents, with the hope to find a clearer and deeper understanding on these challenges. Having a better understanding on the issue may help health education policymakers and healthcare providers in looking for more innovative strategies to overcome such challenges.

In different communities, there are lots of discrepancies between cultures, beliefs, lifestyles, equipment, supportive systems and social-family contexts, which may influence the resident's experiences, attitudes and performance. ${ }^{14}{ }^{15}$ In Iran, as a developing country, the governmental Primary Health Care (PHC) system was developed by the ministry of health to provide the Iranian rural population with a better level of healthcare delivery. In this PHC system, rural health workers (RHWs) are the most vital health service delivery agents. ${ }^{3}$ RHWs, who are working at the rural health houses, are also responsible for educating rural communities based on the national and local health programmes and specific health needs of rural inhabitants. ${ }^{18}$ Every rural health house in Iran is managed by one or two RHWs and the activities of RHWs are supervised by the health professionals at the rural health centres. ${ }^{19}$ Based on the assumption that the relations of these discrepancies to rural health education are not well understood, we performed this study to identify the obstacles and challenges of participating health education programmes in rural communities from the viewpoints of rural residents in Ardebil, Iran.

\section{METHODS}

\section{Study design and participants}

A qualitative study with conventional content analysis approach was used to explore the obstacles to participate in health education programmes in a rural community in Ardabil, Iran during March to October 2016. Twenty-two rural clients who were receiving health services from Ardabil health centres were purposefully invited to participate in the study. Purposeful sampling which is a non-probability sampling method and is widely used in qualitative research for the identification and selection of information-rich participants related to the phenomenon of interest was applied. In addition, maximum variation in terms of age, gender, marital status and education was used. Inclusion criteria were using healthcare services and willingness to participate in the study.

\section{Data collection}

Data were collected through in-depth, semistructured interviews. The second researcher, with considerable experience in conducting qualitative interviews with rural populations, conducted the interviews. An interview schedule including open-ended questions and topic areas developed by the interviewer to be applied while conducting the semistructured interviews. Twenty-two villagers were individually in-depth interviewed in face to face manner to dig deeper down into their perceptions on the obstacles to participate in health education programmes and we held second interview with two participants for more and deep understanding of the participants' view of points. The interviews usually began with a general question, for example, 'Would you please explain your experience of participating in health education programmes?', 'What hinders you to participate in health education programmes?' and 'What factors facilitate or inhibit your participation in health education programmes?' The probing questions were asked based on the participants' responses.

The aim of using voice recorder was explained by the interviewer and all interviews were audio recorded using a voice recorder, anonymously. The time and place of interview sessions were arranged based on the locales convenient to the participants. The aim and process of the study were explained to the participants and they all signed written consent forms. The participants were informed that they had the right to withdraw at any time during the interview. None of participants reused to participate for interview. Each participant was interviewed once for about 40-60 min. The interviews were often performed at the work place or home of the participants.

\section{Data analysis}

To analyse the data, all interviews were transcribed verbatim and the interviews were read several times. The conventional content analysis was started by identifying the units of meaning extracted from the statements. Codes were generated inductively, and the extracted codes were identified as categories based on the differences and similarities. After performing interview with 37 participants, theoretical saturation of the data was achieved and no new code, category and theme emerged in the last two interviews. ${ }^{20}$ MAXQDA software (V.10.0, VERBI Software, Berlin, Germany) was used to manage the textual data.

\section{Data trustworthiness}

The researchers applied the criteria suggested by Guba and Lincoln to evaluate the credibility of the data. ${ }^{21}$ The prolonged engagement with participants during the interview period helped to establish trust and better understanding of the participants. Moreover, the research team checked the interview data and the findings at each step of the study process. Analytic categories, interpretations and conclusions are tested by the participants (member check). In order to account for inter-rater reliability, the first researcher randomly selected and coded one in 
Table 1 Main categories and subcategories of obstacles

\begin{tabular}{ll} 
Categories & Subcategories \\
\hline $\begin{array}{l}\text { Lack of trust to the rural health workers } \\
\text { Lack of acceptability of rural health workers/cultural dissimilarities } \\
\text { Lack of trust in the competence of rural health workers as a health educator } \\
\text { Inability of rural health workers to communicate with villagers }\end{array}$ \\
$\begin{array}{l}\text { Adherence to neighbourhood social networks in } \\
\text { seeking health information }\end{array}$ & $\begin{array}{l}\text { Adherence to neighbourhood social networks in seeking health information } \\
\text { Lack of understanding on the importance of } \\
\text { health education }\end{array}$ \\
\hline
\end{tabular}

seven raw transcripts. We, therefore, checked the themes and ensured that similar themes were deduced by both researchers. All steps followed in the research process were documented by the researchers to provide auditability and dependability of the data.

\section{RESULTS}

This qualitative study provides some insights about the obstacles of participating health education programmes from the viewpoints of villagers in an Iranian rural community. In total, 514 codes and 5 subcategories were extracted from the data. Finally, three categories were described as the main obstacles (table 1): 'Lack of trust to the rural health workers', 'Adherence to neighbourhood social networks in seeking health information' and 'Lack of understanding on the importance of health education'. The categories are extensively discussed as follows:

\section{Lack of trust to the rural health workers}

The villagers' lack of trust to RHWs was reported as a major obstacle to participate in health education programmes. The rural community members for many reasons like cultural dissimilarities and distrust to the health workers' competencies did not participate in rural health education programmes. This concept was explained via three subcategories:

Lack of acceptability of rural health workers/cultural dissimilarities There was a strong feeling in the participants that they were different from those living in the cities, and that they had particular characteristics that were not found among their urban counterparts; hence, many of the villagers preferred to be trained by an indigenous health worker, a member of their own community. They believed that the health workers who are from their own community understood their context better and were more accessible when needed, compared with non-native RHWs. Villagers reported that many health workers were not native and not inhabitants of their village. They also commented that the current health workers occasionally came from urban areas to deliver healthcare services. As they were not indigenous, the villagers could not trust them for receiving health education services. Cultural dissimilarities between the villagers and health workers may disrupt the interactions between them.
'...Some of the health workers come from the city and have different culture and behaviour. So they can't understand our problems.'

Moreover, tribal prejudices were also reported to have impacts on the villagers' participation in health education programmes. The villagers did not accept the educations of non-native health workers who were from the tribes other than their own tribe.

'...There are different tribes in our village. The families from the tribe to which the health worker belongs, will be more cooperative with that health worker; otherwise if the health worker is not belong to their tribe, they won't cooperate.'

Lack of trust in the competence of rural health workers as a health educator

Many villagers believed that the RHWs were not experts and competent enough to implement health education programmes and to deliver health messages properly. Providing the villagers with old and repeating contents, brief and unclear descriptions and infrequent answers to some questions were the reasons for such perceptions. They preferred to receive health messages from clinicians-doctors, nurses and other healthcare providers. They believed that the RHWs were less effective than the general physicians in increasing the knowledge of villagers about health issues.

'The contents noted by them [the rural health workers] are old and out-of-date; but I think the physicians are better because they are expert and their educational contents are based on the up-to-date and scientific contents.'

'The health workers do not have enough ability to teach. They don't explain so properly and their knowledge is not up-to-date.'

Inability of rural health workers to communicate with villagers Many villagers believed that the RHWs usually did not encourage their clients to ask questions during the educational sessions and seldom paid attention to their concerns. They commented that the health workers avoided to discuss their problems in the sessions because they could not manage the discussions or they did not 
have adequate time to discuss. Hence, the learners of health education sessions would be demotivated to participate in such programmes. Furthermore, inappropriate behaviours of some RHWs have made villagers unwilling to participate in the programmes, as participants perceived.

'...I think being friendly with the care givers is important to build a good relationship. Some of the rural health workers are too angry, one does not dare to ask any questions and they do not tend to take part in the classes.'

\section{Adherence to neighbourhood social networks in seeking health information}

According to participants, people in rural communities are affected by the opinions of relatives and neighbourhood. They prefer to seek health information from other villagers. As a result, in the case that their relatives or peers consider health information as a valuable thing, then the villagers easily admit their opinions. In other words, they consult with neighbourhood social networks before they visit the RHWs. When they realise no improvement in their conditions, they decide to go to the rural health houses and to participate in the programmes.

'...if I have a health problem, I consult with my neighbours or family members, at first. If their recommendations couldn't help me so I go to the rural health house.'

\section{Lack of understanding on the importance of health education} The villagers reported that the significance and necessity of health education was not still well perceived by the rural inhabitants. They were believed on a lack of understanding on the importance of health education programmes.

\section{Giving low priority to health needs}

Based on the participants' beliefs, lack of valuing to health promotion and disease prevention among villagers was another obstacle to participate in rural health education programmes. They commented that many rural community members have not perceived the benefits of applying health-related materials and preventive actions in their everyday lives. Curative activities and medical services use were the key reasons for them to be motivated to refer rural health houses.

'...in the village, people don't care about their health. They are still not aware about the role of prevention. Just whenever getting sick go to the rural health house to get medical services.'

\section{Too emphasis on livelihood needs}

The participants reported that the villagers came to the health houses and wanted to leave there in a hurry. As participants perceived, their hastiness was due to the stress caused by the lack of time and high level of workload.
They commented that in the villages there was very much focus on getting the job done, so the villagers wanted to quickly go back home or work place to do their job. Therefore, they did not so care about participating health education programmes and learning activities.

'Our workloads are too high. Our job at the farmlands keeps us busy all the time; we have too many duties which are really stressful.'

\section{DISCUSSION}

Our data indicated lack of trust as a key contextual obstacle among rural inhabitants to accept RHWs. Trust seems to play a detrimental role in the relationships between healthcare providers and clients, particularly in traditional communities. Many villagers in the present study indicated cultural dissimilarities between the RHWs and the rural-dwelling people, who were doubtful to RHWs' competencies to educate the villagers. As they commented, the rural inhabitants preferred to visit physicians, registered midwives or health professionals in the health centres instead of referring to the RHWs in the health houses. Several previous studies have reported that the success of health interventions relies on positive and trustful relationships between healthcare workers and clients. $^{22} 23$ Although many researchers have emphasised good interactions between the clients and the healthcare providers as a key factor for successful uptake of health services, ${ }^{24} 25$ the magnitude of trust within such relationships is often undermined in health systems. ${ }^{26}$ Singh et al (2015) also reported that trust improvement may lead to better contribution of CHWs to the clients during educational programmes, which may in turn promote maternal and child health outcomes in low-income and middle-income countries. ${ }^{27}$ Additionally, clients' comfort with the health workers and medical doctors, physician-patient relationships based on trust and mutual respect, behaviour and approach of health professionals and health awareness were identified as factors determining the clients' trust to healthcare systems. ${ }^{28} 29$

Adherence to neighbourhood social networks in seeking health information was another finding of our study, which may lead to pay less attention to the RHWs recommendations. Many villagers reported to act in their own social relationship environment based on relatives and neighbours' recommendation instead of health professionals. Neighbourhood social environment may influence the pattern of adherence in traditional communities. People in rural communities often show the importance of maintaining their rural atmosphere, remaining family friendly and having closed relationship with fellow-citizens. ${ }^{30}$ In rural communities, compared with urban communities, it seems that the family members have more influence on each other, and there is a higher level of peer pressure on the members to behave in a certain way. Also, in such traditional rural areas, there are accepted ways to behave in a particular way, which may be directly associated to the culture of society. 
Moreover, attitudes and desires in such areas are strongly influenced by the society's culture. ${ }^{31}$ Although cultures and social structures within communities are always changing, there may be features in the societies and cultures that impede changes in health information seeking behaviours. Findings of Bowen and Wretman (2014) indicated that rural neighbourhoods may operate as both a microsystem and an exosystem for children, with direct contagion effects on their behaviours and indirect social control effects through parenting practices. ${ }^{32}$ Although not directly associated to the villagers' practice of participating health education programmes, this claim highlights the role of rural neighbourhoods as a micro-exosystem on the mode of participation in health education programmes within rural communities. McCann et al (2014) postulated that people in rural areas, compared with those in urban areas, experienced better family support by living as parts of two or three generation households and older rural dwellers were also less likely to enter care homes, so the role of rural neighbours and relatives in providing more informal care to villagers is pivotal. ${ }^{33}$

Many participants reported lack of understanding among villagers on the importance of health education. They believed that the health needs are not a priority for the villagers compared with the livelihood needs and economic issues, which ail many inhabitants in such rural areas. For the villagers, addressing the livelihood needs was more important than seeking health information. A majority of the rural people, as underprivileged residents, engage in full time farming activities and our findings indicated that the villagers prefer to enact in their peasant activities instead of spending their times to seek for health information.

Seeking for health information would not be more important when socioeconomic needs are not met. It was previously reported that a large number of rural people are involved in agriculture and its related activities, which make them too busy ${ }^{33}$ to participate in health programmes. These findings suggest considering the rural people's livelihood needs while planning rural health education programmes. In fact, in global terms, poverty is predominantly a rural phenomenon and a great proportion of underprivileged people throughout the world live in rural areas. ${ }^{34}$ It is also postulated that education level is often low in disadvantaged rural areas, and the low level of education may contribute to low employment rates, which may consequently increase the rate of poverty. Such poverty in disadvantaged rural areas may negatively affect the chance of rural people for receiving high-quality education ${ }^{35}$ and health information as well. Low rates of engagement in early childhood services, ${ }^{36} 37$ distance from educational facilities and low quality of education due to infrastructural and staff qualification reasons ${ }^{37}$ are also among the reasons for not receiving quality health education. In a study conducted in rural Sri Lanka, ${ }^{38}$ lifestyle and time management as well as environmental and social factors (like social embarrassment and giving priority to household activities other than health behaviours) were reported as the factors that limited physical activity among the residents. Another study in the southwest of $\operatorname{Iran}^{39}$ showed economic and social barriers as the factors that impede the rural diabetic patients to attend in scheduled appointments. Similarly, maternal health services in rural Cambodia were under influence of financial barriers, which may be due to high enactment in peasant activities. ${ }^{40}$

As a limitation in the present study, the small sample size may be noted, which is due to the qualitative design of the study. The selection of participants only from some villages of Ardabil province may have limited the representativeness of the sample and generalisability of the results. We also did not triangulate the results with quantitative approaches.

\section{CONCLUSION}

The villagers described multiple obstacles to effectively participate in rural health education programmes. Lack of trust to the RHWs, adherence to neighbourhood social networks in seeking health information and lack of understanding on the importance of health education influenced the villagers' participation in rural health education programmes. Our data highlighted the need for doublefaced strategies to address the issue, which should focus primarily on enhancing mutual trust between the RHWs and villagers, developing the communication skills of the RHWs, minimising the impacts of personal and contextual factors (like time and financial cost), and developing community-based education programmes to promote health information seeking behaviours among villagers. Although such strategies may hold promise, the effects of targeted and tailored strategies on promoting villagers' participation in rural health education programmes remain to be tested empirically.

\section{Key points}

What is this research focused on exploring, validating, or solving?

- Because of numerous problems to reach rural communities, the aim of present study was to explore how rural-dwelling populations in Iranian context explain obstacles to participate in rural health education programs.

What conclusions did this research draw through design, method, and analysis?

- We concluded that based on the emerged findings it is needed for double-faced strategies to address the issue, which should focus primarily on enhancing mutual trust between the RHWs and villagers, and next, developing community-based education programs.

What is the value, meaning and impact of your research? Is there any follow-up study based on this research?

- The most important value of this study was to look obstacles of health education programs based onthe view points of Iranian rural inhabitants which will guide us for tailoring effective health education programs especially for rural inhabitants based on their own context. 
Acknowledgements This study was a part of a large project supported by Tabriz University of Medical Sciences. The research team wishes to thank all the participants.

Funding This study was a part of a large project supported by Tabriz University of Medical Sciences Postgraduate Award for her PhD degree. This research received no specific grant from any funding agency in the public, commercial, or not-forprofit sectors.

Disclaimer There is no any figures or tables from another publication in this paper. Competing interests None declared.

Patient consent for publication Not required.

Ethics approval Ethical approval for the study was provided by ethics committee in Tabriz University of Medical Sciences (Ethics Code: IR.TBZMED.REC. 1395.285). Informed consent form was obtained from all the participants before the interviews.

Provenance and peer review Not commissioned; externally peer reviewed.

Open access This is an open access article distributed in accordance with the Creative Commons Attribution Non Commercial (CC BY-NC 4.0) license, which permits others to distribute, remix, adapt, build upon this work non-commercially, and license their derivative works on different terms, provided the original work is properly cited, appropriate credit is given, any changes made indicated, and the use is non-commercial. See: http://creativecommons.org/licenses/by-nc/4.0

\section{REFERENCES}

1. Strasser R, Kam SM, Regalado SM. Rural health care access and policy in developing countries. Annu Rev Public Health 2016;37:395-412.

2. Prout S, Lin I, Nattabi B, et al. 'I could never have learned this in a lecture': transformative learning in rural health education. Adv in Health Sci Educ 2014;19:147-59.

3. Hempel S, Gibbons MM, Ulloa JG. Rural health care workforce: a systematic review. VA ESP Project \#05-226. Washington DC: Department of Veterans Affairs, 2015.

4. National Rural Health Association, 2017. What's different about rural health care? Available: http://www.rural health web.org/go/left/aboutrural-health/what-s-different-about-rural-health-care [Accessed 6 Mar 2017].

5. Douthit N, Kiv S, Dwolatzky T, et al. Exposing some important barriers to health care access in the rural USA. Public Health 2015;129:611-20.

6. Weinhold I, Gurtner S, Sebastian Gurtner G. Understanding shortages of sufficient health care in rural areas. Health Policy 2014;118:201-14.

7. McQueen DV. Global Handbook on non-communicable diseases and health promotion, science +Business media, LLC. Springer, 2013: 73.

8. Ardalan A, Mowafi $\mathrm{H}$, Malekafzali Ardakani $\mathrm{H}$, et al. Effectiveness of a primary health care program on urban and rural community disaster preparedness, Islamic Republic of Iran: a community intervention trial. Disaster Med Public Health Prep 2013;7:481-90.

9. Shakiba M, Haghdoost A, Majdzadeh S. The application of geographical information system in explaining spatial distribution of low birth weight; a case study in North of Iran. Iran J Med Sci 2015;33:220-5.

10. World Health Organization. Global strategy for health for all by the year 2000. 3. Geneva: WHO, 1981.

11. Sadrizadeh B. Primary health care experience in Iran. Iran Red Crescent Med J 2004;7:79-90.

12. Manenti A. Health situation in Iran. Med J Islam Repub Iran 2011;25:1-7.

13. Abbaszadeh A, Eskandari M, Borhani F. Changing the care process: a new concept in Iranian rural health care. Asian Nurs Res 2013;7:38-43.

14. Larry G, Linnae H, Gail B. Dabney: rural healthy People 2010: identifying rural health priorities and models for practice. J Rural health 2002;18:9-14.

15. Kronfol NM. Access and barriers to health care delivery in Arab countries: a review. Easter Mediterr Health J 2012;18:1239-46.

16. Salehi Zalani G, Bayat M, Shokri A, et al. Affecting factors on the performance of community health workers in Iran's rural areas: a review article. Iran J Public Health 2016;45:1399-410.

17. Clarke JL, Bourn S, Skoufalos A, et al. An innovative approach to health care delivery for patients with chronic conditions. Popul Health Manag 2017;20:23-30.
18. Azizi F. The reform of medical education in Iran. Med Educ 1997;31:159-62.

19. Couper I. Rural primary health care in Iran. South African Family Practice 2004:46:37-9.

20. Graneheim UH, Lundman B. Qualitative content analysis in nursing research: concepts, procedures and measures to achieve trustworthiness. Nurse Educ Today 2004;24:105-12.

21. Guba E, Lincoln Y. Naturalistic inquiry Nol. 75. Beverly Hills, CA: Sage, 1985.

22. Gilson L. Trust and the development of health care as a social institution. Soc Sci Med 2003;56:1453-68.

23. Greeff M, Phetlhu R, Makoae LN, et al. Disclosure of HIV status: experiences and perceptions of persons living with HIV/AIDS and nurses involved in their care in Africa. Qual Health Res 2008;18:311-24.

24. Mishra A. 'Trust and teamwork matter': Community health workers experiences in integrated service delivery in India. Glob Public Health 2014;9:960-74.

25. Balcazar $\mathrm{H}$, Rosenthal EL, Brownstein JN, et al. Community health workers can be a public health force for change in the United States: three actions for a new paradigm. Am J Public Health 2011;101:2199-203.

26. Grant M, Wilford A, Haskins L, et al. Trust of community health workers influences the acceptance of community-based maternal and child health services. Afr J Prim Health Care Fam Med 2017;9:1-8.

27. Singh D, Cumming R, Negin J. Acceptability and trust of community health workers offering maternal and newborn health education in rural Uganda. Health Educ Res 2015;30:cyv045-58.

28. Gopichandran V, Chetlapalli SK. Dimensions and determinants of trust in health care in resource poor settings--a qualitative exploration. PLoS One 2013;8:e69170.

29. Ware VA, 2013. Improving the accessibility of health services in urban and regional settings for Indigenous people. Available: https://www.aihw.gov.au/reports/indigenous-australians/improvingthe-accessibility-of-health-services-in-urban-and-regionalsettings-for-indigenous-people/contents/table-of-contents [Accessed 30 Aug 2018].

30. Daniels TL, Keller JW, Lapping MB. The small town planning Handbook. 3rd edn. Chicago, IL: American Planning Association Planners Press, 2007.

31. Karan A, Chapman C, Galvani A. The influence of poverty and culture on the transmission of parasitic infections in rural Nicaraguan villages. J parasite res 2012.

32. Bowen NK, Wretman CJ. Rural neighborhoods and child aggression. Am J Community Psychol 2014;54:304-15.

33. McCann M, Grundy E, O'Reilly D. Urban and rural differences in risk of admission to a care home: a census-based follow-up study. Health Place 2014;30:171-6.

34. Dorward A MJ, 2018. Rural livelihoods: importance and definitions. Available: http:// www.soas.ac.uk/cedep-demos/000_ P535_SRL_K3736-Demo/unit1/page_08.htm [Accessed 28 Aug 2018].

35. Zimmerman EB, Woolf $\mathrm{SH}$, Haley A. Understanding the relationship between education and health: a review of the evidence and an examination of community perspectives. Rockville, MD: Agency for Healthcare Research and Quality, 2015.

36. European Commission, 2018. Poverty and social exclusion in rural areas. Final study report. Luxembourg, office for official publications of the European communities. Available: http://ec. europa.eu/social/main.jsp?catld [Accessed 28 Aug 2018].

37. World Health Organization, 2010. Rural poverty and health systems in the WHO European region. Copenhagen, who regional office for Europe. Available: http:// ww.euro.who.int/_data/assets/pdf_file/ 0019/130726/e94659.pdf [Accessed 20 Aug 2018].

38. Medagama A, Galgomuwa M, infrastructure Lof. Lack of infrastructure, social and cultural factors limit physical activity among patients with type 2 diabetes in rural Sri Lanka, a qualitative study. PLoS One 2018;13:e0192679.

39. Heydarabadi AB, Mehr HM, Nouhjah S. Why rural diabetic patients do not attend for scheduled appointments: Results of a qualitative study. Diabetes \& Metabolic Syndrome. Clin Res Rev 2017;11:S989-S995.

40. Matsuoka S, Aiga H, Rasmey LC, et al. Perceived barriers to utilization of maternal health services in rural Cambodia. Health Policy 2010;95:255-63. 